\title{
A nature-inspired approach to reactor and catalysis engineering Marc-Olivier Coppens
}

\begin{abstract}
Mechanisms used by biology to solve fundamental problems, such as those related to scalability, efficiency and robustness could guide the design of innovative solutions to similar challenges in chemical engineering. Complementing progress in bioinspired chemistry and materials science, we identify three methodologies as the backbone of nature-inspired reactor and catalysis engineering. First, biology often uses hierarchical networks to bridge scales and facilitate transport, leading to broadly scalable solutions that are robust, highly efficient, or both. Second, nano-confinement with carefully balanced forces at multiple scales creates structured environments with superior catalytic performance. Finally, nature employs dynamics to form synergistic and adaptable organizations from simple components. While common in nature, such mechanisms are only sporadically applied technologically in a purposeful manner. Nature-inspired chemical engineering shows great potential to innovate reactor and catalysis engineering, when using a fundamentally rooted approach, adapted to the specific context of chemical engineering processes, rather than mimicry.
\end{abstract}

\section{Address \\ Howard P. Isermann Department of Chemical and Biological Engineering, Rensselaer Polytechnic Institute, 110 8th Street, Troy, NY 12180, USA \\ Corresponding author: Coppens, Marc-Olivier (coppens@rpi.edu)}

Current Opinion in Chemical Engineering 2012, 1:1-9

This review comes from a themed issue on

Reaction engineering and catalysis

Edited by Theodore Tsotsis

2211-3398/\$ - see front matter

Published by Elsevier Ltd.

DOI 10.1016/j.coche.2012.03.002

\section{Introduction}

Nature-inspired engineering researches the fundamental mechanism underlying a desired property or function in nature, most often in biology, and applies this mechanism in a technological context. In the context of chemical engineering, we call this approach: nature-inspired chemical engineering (NICE) [1].

Application of biological mechanisms to a non-physiological context in reaction engineering requires adaptations, because the relevant time scales and available building blocks are different. Also, we are able to manipulate parameters such as temperature and pressure, which are much less tunable in biology. Hence, like in an abstract portrait, essential aspects of the subject are preserved, but not literally, emphasizing those features that serve a desired purpose. Such features underpin the rational design of an artificial structure that uses the same fundamental mechanism as the natural system. The ultimate implementation is assisted by theory and experimentation. NICE aims to innovate, guided by nature, but it does not mimic nature, and should be applied in the right context.

Emphasizing reactor and catalysis engineering, we illustrate how mechanisms used in biology to satisfy complicated requirements, essential to life, are adapted to guide innovative solutions to similar challenges in chemical engineering. These mechanisms include: (1) use of optimized, hierarchical networks to bridge scales, minimize transport limitations, and realize efficient, scalable solutions; (2) careful balancing of forces at one or more scales to achieve superior performance, for example, in terms of yield and selectivity; (3) emergence of complex functions from simple components, using dynamics as an organizing mechanism. Figure 1 presents an overview.

In this way, NICE complements an ongoing revolution in bioinspired chemistry and materials science $\left[2^{\bullet \bullet}, 3^{\bullet \bullet}, 4-6\right]$, which already sees applications in, for example, enzymemimics and antibody-mimics for catalysis [7-10] and in artificial photosynthesis $\left[11^{\bullet}, 12^{\bullet}, 13-15\right]$. These applications implement essential mechanistic steps of the biological model system at molecular and supramolecular scales. Hierarchically structured bionanocomposites have superior properties by synergy, unmatched by their individual components, inspiring novel material designs.

As we now illustrate, nature has more to offer to reaction engineering when considering larger length scales and the time domain. In addition, the manipulation of force balances as an organizing mechanism merges bioinspired chemistry, chemical and materials engineering.

\section{Hierarchical transport networks}

Transport is crucial to living systems, and to reaction engineering alike. Trees and mammalian physiological networks share common architectural traits that endow them with vital properties. The vascular and respiratory networks have a branched, hierarchical architecture that is fractal between macroscopic and mesoscopic length scales, having features that look similar under repeated magnification $\left[16^{\bullet \bullet}, 17\right]$. At those scales, convective flow is the dominating transport mechanism and the channel walls are impermeable. On the contrary, channels are 
Figure 1

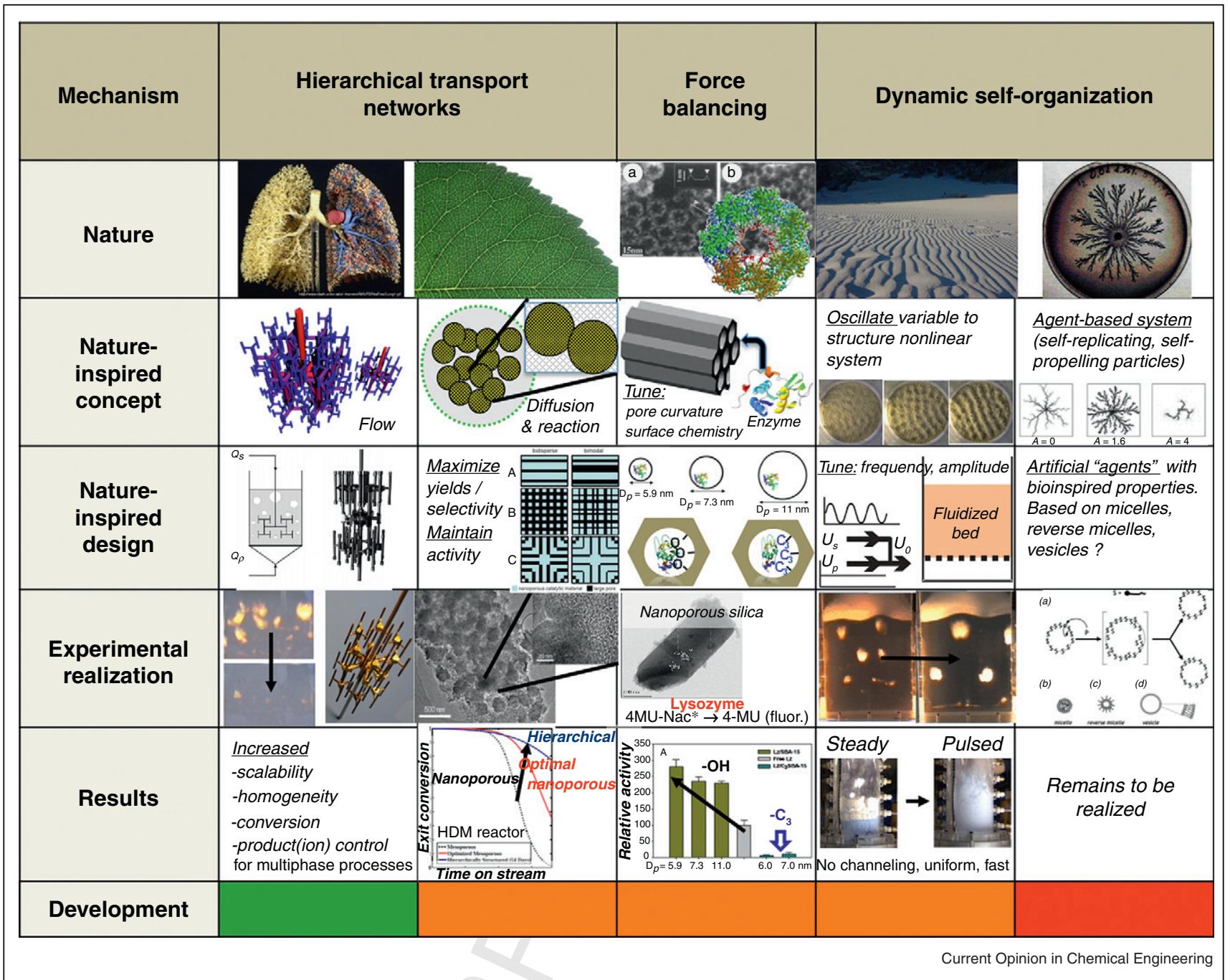

Overview of nature-inspired chemical engineering, as applied to catalysis and reactor engineering, from observation to concept, design and realization. The bottom row indicates the stage of development, from green (ready for industrial development) to red (early-stage). All images personal, and from $\left[21^{\bullet}, 23^{\bullet}, 46^{\bullet}, 57^{\bullet}, 81,92,95^{\bullet}, 102\right]$, except on top row: lung [103]; leaf [104]; GroEL heptamers [53]; bacterial colony on Petri dish [105].

almost uniformly sized at mesoscopic to microscopic length scales, approaching those of individual cells. In capillaries or in the acini of the lung, exchange occurs via the cell walls, and diffusion is the principle transport mechanism. This is most efficient, costing no metabolic energy [18 $\left.{ }^{\bullet}\right]$. Indeed, the transition between biological circulatory networks and networks of exchanging channels frequently corresponds to a Péclet number around 1. Furthermore, as discussed below, such architectures are optimal in several other ways that would benefit chemical engineering applications.

The fractal architecture of the upper respiratory tract, the arterial network and tree crowns connects multiple microscopic elements to a single macroscopic feeding/collection point (trachea, heart, stem). This occurs via equal hydraulic path lengths that provide equal transport rates to and from the cells. Cell size is remarkably constant across species, despite considerable differences in size between organisms. Feeding more cells occurs via trees with a larger number of branching generations. The fractal geometry of biological transport networks facilitates scale-up, by preserving cell access and function irrespective of size. Achieving uniform access and macroscopically homogeneous operation are chemical engineering challenges as well. This insight has led to the construction of fractal distributors and injectors for multiphase separation, mixing and reaction processes $\left[19,20^{\bullet}, 21^{\bullet \bullet}\right]$.

Figure 2 includes an example of a two-dimensional $(D=2)$ fractal distributor from our laboratory, produced 
Figure 2

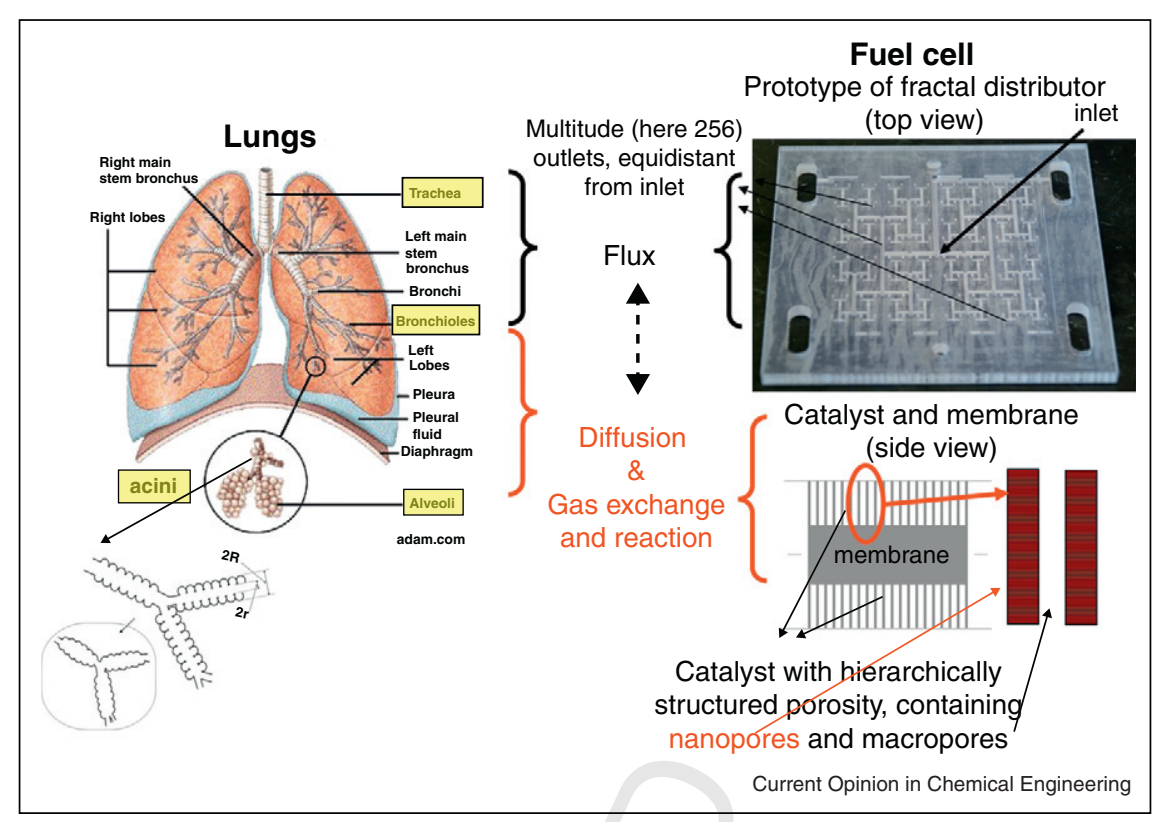

Fuel cell design guided by the architecture of the lung, and the associated physical mechanisms.

by stereolithography, for a PEM fuel cell design inspired by the structure of the lung. Fluid entering the distributor through a single inlet flows through the branching channels, and ultimately leaves the distributor through a square array of outlets, which are hydraulically equidistant from the inlet. Thus, the space under the distributor is accessed uniformly. This fractal distributor could uniformly feed air over the catalytic layer of a PEM fuel cell, as well as collect water, circumventing non-uniformity issues of serpentine and other channel geometries [22]. Such structures could also homogeneously feed high-throughput setups, or uniformly heat or cool surfaces. They could be integrated into microfluidic devices; already common in multi-channel microreactors is a binary tree, based on $n$ times repeated Y-branching, to serve a one-dimensional array of $2^{n}$ channels $(D=1)$.

In nature, the fractal dimension, $D$, depends on the transport network. The respiratory network of a lung fills space, hence $D=3$. In other cases, as for botanical trees, the structure fills less than three-dimensional space, but is more than area-filling, therefore $2<D<3$. For example, splitting all branch tips of a self-similar tree into 6 new branches that are half as extended leads to a tree with $D=\log 6 / \log 2 \approx 2.6$. Such is the case for the fractal injector shown in Figure $3\left[21^{\bullet \bullet}, 23^{\bullet}\right]$.

Figure 3

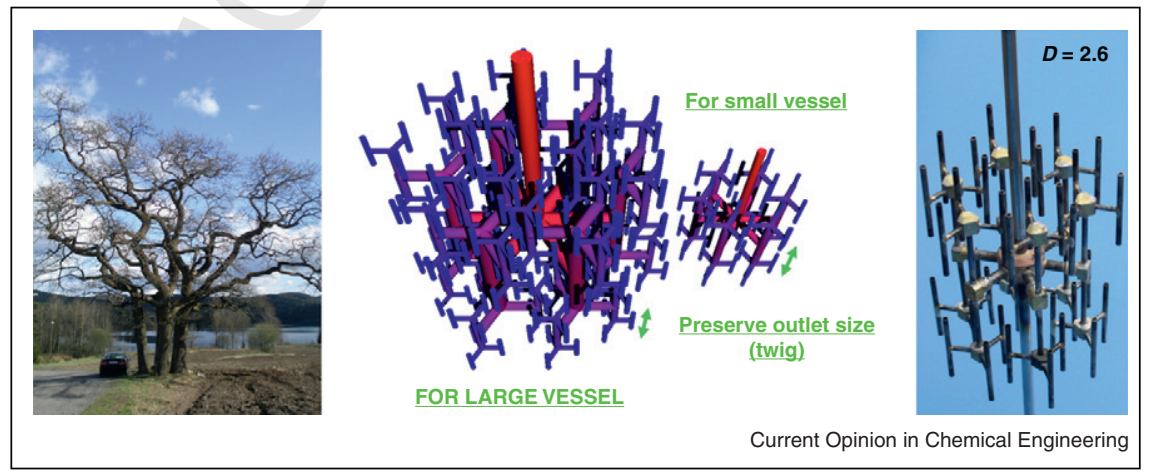

Fractal injector for multiphase reactors, guided by the scaling architecture of tree crowns. 
Submerged in a fluidized bed, this fractal injector uniformly distributes gas throughout the reactor. Maintaining at least minimum fluidization via a bottom distributor plate, extra gas injected via the fractal injector, directly into the emulsion phase, promotes micromixing around the branch tips. This increases mass and heat transfer, and improves gas-solid contact, because of kinetically delayed bubble formation. For multiphase reactors, the complex hydrodynamics are a major hurdle in scale-up [24]. A fractal injector facilitates scale-up: like in a lung or tree, the sizes of the outlets and the distances between outlets are maintained in larger reactors, by increasing the number of branching generations. The optimal distribution of outlets and value of $D$ depend on mixing characteristics and reaction times $\left[21^{\bullet \bullet}\right.$. Utilizing a fractal injector can bring the overall reactor behavior closer to plug flow, which is often advantageous to increase conversions and, depending on the kinetics of the competing reactions, selectivity $\left[23^{\circ}\right]$. For applications at high temperatures and large scales, the hierarchical structure lends itself well to modular, metallic construction.

Another remarkable feature of many scaling, anatomical trees is that they are near optimal from the point of view of flow resistance, thermodynamics, and robustness over time and over a range of operating conditions. Often, this is tied to the particular geometric progression of branch lengths and radii, and the minimum length scale of the scaling regime. Such observations date back almost a century, when Murray showed that, for the vascular network, mechanical resistance and the cost of maintenance of blood in the body are minimal thanks to a branched structure in which the sum of the cubes of the diameters of daughter branches is equal to the cube of the diameter of the parent branch $[25,26]$. West et al. $\left[27^{\bullet}, 28,29\right]$ further postulated that space-filling, biological circulatory networks relate to mechanical and thermodynamic optimality, and are the cause behind Kleiber's allometric scaling law (energy dissipation proportional to body mass to the power). Bejan and co-workers $\left[30^{\bullet \bullet}, 31\right]$ introduced a thermodynamic theory to derive optimal architectures satisfying various criteria, for example, maintaining uniformity, reducing flow resistance [32] or minimizing the maximum temperature of a surface. Using this 'constructal theory' remarkable parallels between trees in nature and engineering were found. Frequently, optimality corresponds to architectures that realize equipartition of entropy production, as was discovered for the lung [33]. Tondeur and Luo [34,35] applied constructal theory to distributors that compromise costs related to pressure drop, viscous dissipation, and hold-up volume.

When diffusion is the dominating transport mechanism, the architecture of biological transport networks changes from fractal, scaling, to uniform, non-scaling, in particular when exchange processes via the walls occur, as in acini and leaves. Translation to catalysis engineering requires care, as objectives and constraints might differ. For example, reactor-engineering requirements often determine minimum catalyst particle size, resulting in possible diffusion limitations. Other criteria are problem dependent: minimizing costly catalytic component to achieve a certain yield, maximizing conversion, mitigating effects of deactivation, achieving a particular product distribution, and so on. We do not review this subject in depth here, but refer to Ref. [36]. Simulation relies on a range of modeling approaches [37], which are increasingly multiscale $[38,39]$. Recent possibilities to control pore network properties at multiple length scales via new synthesis methods $\left[40,41,42^{\bullet}\right]$ should be accompanied by theoretical optimization. If the intrinsic catalytic activity per unit nanoporous catalyst is kept constant, as in zeolites or catalytic clusters supported on a mesoporous carrier, then virtually no benefit is gained from a broad macro/mesopore size distribution to increase activity $\left[43,44^{\circ}\right]$, increase stability $\left[45,46^{\circ}\right]$ or control selectivity [47]: optimal porosity and optimal average macro/mesopore size are the main parameters. Other criteria may lead to different optima [48,49]. Most important is that the hierarchically structured catalyst consists of nanoporous domains or grains without local diffusion limitations, interspersed by larger pore channels of optimized size. Again, this matches physiology: cells of the same type are of the same size, and interspersed by capillaries of more or less uniform size that transport nutrients and remove waste products by diffusion.

The ability to bridge scales and efficiently couple transport and reaction processes by nature-inspired design promotes process intensification [50]. This is illustrated by Figure 2, showing how the structure of the lung inspires the design of a PEM fuel cell, with the aim to drastically reduce the required amount of expensive $\mathrm{Pt}$ catalyst to achieve a desired power density, facilitate water management, maintain uniform operation, increase robustness, and facilitate scale-up.

\section{Force balancing}

From the DNA double helix to virus capsids, biology is replete with supramolecular assemblies that self-organize from molecular and ionic components via balanced, noncovalent interactions [3,51]. Hierarchically structured materials can be synthesized using biological templating or mechanisms used in biomineralization and biological layer-by-layer assembly $\left[2^{\bullet \bullet}, 4,5,6,52\right]$. Their superior properties are not trivially inferred from those of the components.

Catalysis could also benefit from optimized force balancing by implementing nano-confinement effects observed in biology. A case in point are molecular chaperones, which prevent aggregation of a number of proteins in crowded cells, and assist proteins to assume 
their native conformation in vivo. The GroEL/GroES system in E. coli contains protein heptamers surrounding a nano-cage with a diameter of $\sim 4.5 \mathrm{~nm}$ [53]. Steric confinement helps proteins fold, thanks to periodic electrostatic interactions that result from a negatively charged internal surface during the time of folding. While the details are complicated, the GroEL/GroES system informs us on how different degrees of steric confinement, hydrophobicity, modulated surface charge and confined water content could be employed to tune protein stability $\left[54,55,56^{\bullet}\right]$.

This insight can be applied to design catalysts consisting of nanostructured porous materials, such as SBA-15 silica, with constant, but tunable mesopore diameter, and enzymes immobilized on the pore surface. We recently observed that the catalytic activity of positively charged lysozyme or myoglobin, electrostatically adsorbed on the negatively charged pore surface of SBA-15, increased several times with respect to that of the free enzyme in aqueous solution, with minimal leaching $\left[57^{\circ}\right]$. The highest activity was measured in SBA-15 with the narrowest pores. This smallest pore diameter $(\sim 6 \mathrm{~nm})$ barely exceeds the dimensions of lysozyme $(3.0 \mathrm{~nm} \times 3.0 \mathrm{~nm} \times 4.5 \mathrm{~nm})$ and myoglobin $(2.9 \mathrm{~nm} \times 3.6 \mathrm{~nm} \times 6.4 \mathrm{~nm})$, and approximates the cage diameter of GroEL. Confinement in nanopores not only allows us to tune catalytic activity, but it also facilitates enzyme recovery, may prevent denaturation, and improves thermal and environmental stability [58 ${ }^{\bullet}$. Spectroscopic studies indicated that the balanced electrostatic-steric interactions prevent unfolding, by stabilizing the protein's native conformation $\left[57^{\bullet}\right]$. On the contrary, when the silica surface was functionalized with propyl groups, rendering it hydrophobic, the protein's conformation changed considerably, and activity dropped.

Computer simulations of polypeptides in nano-confining spaces provide clues on how confinement affects enzyme structure $\left[59-61,62^{\bullet}, 63\right]$. The structure of confined water around enzymes in nanopores differs from that of bulk water, so that water-mediated interactions often affect the free energy landscape, and hereby enzyme stability $\left[64,65^{\circ}\right]$.

Electrostatics, steric confinement, hydrophobicity, and H-bonding all influence the activity and stability of enzymes. Mechanistic understanding of biological pores guide the design of artificial catalytic systems. In turn, studies of model nanostructured catalysts with tunable characteristics, like enzymes in functionalized SBA-15, advance our insight into biological systems.

\section{Dynamic self-organization}

Living systems are dynamic. A third opportunity for NICE lies in the time domain, essential to biological processes, to generate desired spatiotemporal behavior by synergy. Sometimes called 'emergence of complexity' $\left[66,67^{\bullet}, 68^{\bullet \bullet}\right]$, robust properties collectively emerge from individual elements with much more basic functions. Dynamic structuring is rarely consciously applied in chemical engineering, and hardly in an optimal way. Nanotechnology and microtechnology opens avenues to realize $\left[69^{\circ}\right]$ what was originally only conceivable mathematically or on crystal surfaces $\left[70,71,72^{\bullet}, 73\right]$. Learning how versatile, adaptable patterns emerge from biological system dynamics might guide new modes of reactor operation, and the design of new catalytic materials.

Periodic perturbation of a nonlinear system may form simple patterns $\left[74^{\bullet \bullet}\right]$. For example, the actions of water or wind create regularly spaced ridges on sandy beaches and dunes. Likewise, patterns develop when vibrating a plate covered by a thin layer of solid particles [75], an example of the rich, collective behavior of granular matter [76,77]. Energy is constantly provided to a nonlinear system, in which dissipation leads to pattern formation-an example of dynamic self-assembly [78 ${ }^{\bullet \bullet}$.

Hence, the idea to structure gas-solid fluidized beds by pulsing them with a periodically fluctuating gas flow, superimposed on a constant flow to maintain minimum fluidization $\left[7^{\circ}\right]$. In a laterally thin, quasi-2D bed, this led to a hexagonally ordered array of rising bubbles, with a frequency that was half that of the pulsation, within a range of frequencies of a few $\mathrm{Hz}$. Fluidized beds have complex hydrodynamics, which van den Bleek et al. [80] described as deterministically chaotic. By pulsing the gas, fluidization is more uniform, and channeling and clumping are prevented. This improves the performance of fluidized bed combustion and drying $[81,82]$. Periodically perturbing the gas in a fluidized bed can suppress chaos by 'phase locking' [83], however the periodicity of the bubble pattern is remarkable [79 ${ }^{\circ}$. In pulsed $3 \mathrm{D}$ beds, we observed patterns similar to those for vibrated granular media $\left[79^{\bullet}, 84\right]$. While not as high as in quasi-2D beds, the patterns persisted in deeper beds than vibrated granular matter, due to less frictional dissipation. Interestingly, computational fluid dynamics (CFD) has not yet reproduced these experimental patterns, even though some level of structuring has been demonstrated [85]. We suggest that reproducing these patterns should be an interesting fingerprint to test CFD codes.

Other ways to stabilize a nonlinear dynamic system use closed-loop control. Hudson and co-workers [86] recently used (de-)synchronization methods to tune the collective response from weakly interacting rhythmic components, similar to those in biological systems, and applied them to control an electrochemical reaction system.

Bacterial communities present us with one of the most exciting examples of a dynamically self-organized system. Bacteria interact with their environment and each other, but are also self-propelling and self-replicating. Together, 
they form complex communities that are more robust and adaptive than individual bacteria $\left[8^{\circ}\right]$. When starved, they may self-organize into self-similar patterns $[88,89]$, reminiscent of diffusion-limited aggregation (DLA) and other fractal aggregates seen in non-living systems [90], but their adaptive, collective behavior is richer. Bacterial communities attract interest in the context of biofilm research and engineering for chemical production [91]. They also stand model for other self-organizing systems, for example, in sociology [89].

Within the context of NICE, we see an opportunity to design artificial catalytic systems from elements that are not necessarily biological, yet use key aspects of bacterial communities. Building upon von Neumann's pioneering work on self-replicating automata, agent-based methods are ideally suited to explore the diversity of such bioinspired systems [92]. 'Agents' have internal states, can store energy and information, interact, sense and respond to their environment $\left[66,93^{\circ}\right]$. Luisi and co-workers $\left[94,95^{\circ}\right]$ have explored the use of synthetic self-reproducing vesicles as minimal cells. This leads us to postulate that adaptive, self-replicating, internally or externally driven catalytic systems could be implemented, even based on purely artificial components.

Cooperative phenomena result from many interactions in a network of synergistic links $\left[68^{\bullet \bullet}\right]$. However, not all nodes and links are equally relevant. Some are more important, sensitive or robust than others. Collective behavior acts as an evolving, complex network [96100], frequently with universal features, like scaling, clustering, and modularity $\left[101^{\bullet}\right]$.

It would seem that insights in biological systems, reaction pathways and social networks, gained from topology, graph theory and information theory, could be useful not only in synthetic biology and process control, but also in generating more robust and adaptive bioinspired catalytic systems.

\section{Conclusions}

What makes biological organisms especially interesting from the viewpoint of chemical reaction engineering is that efficiency, scalability, robustness, and adaptability are quintessential to both, yet nature uses an arsenal of tools barely touched in engineering. In the context of recent progress, we have provided a personal view on how nature's hierarchical transport networks, force balancing and collective dynamics might be employed in reaction engineering design. At present, some fundamental mechanisms that serve biology so well are slowly permeating materials science and chemistry. However, they are scarcely applied in chemical reactor design and catalysis engineering.

Perhaps this is because we are rooted in an atomistic, bottom-up way of thinking that has helped us tremen- dously over the past century, yet we are confronted with a seemingly insurmountable gap between increasing nanoscale insights and capabilities, where rational design becomes a reality, and applications at the scale of macroscopic production, where empiricism seems inevitable.

Our examples show that this gap could be bridged by rational design principles based on nature-inspired chemical engineering, with the potential to transform what is too often and incorrectly considered a mature field, hereby helping to create sustainable processes. Such designs unite the atomistic and the holistic, using efficient mechanisms in natural systems as guidance for artificial designs-but not as models that are to be slavishly copied as automatically superior, without regard for context.

\section{Acknowledgements}

MOC gratefully acknowledges support from the National Science Foundation via CBET-0967937, DGE-0504361 and 0333314, as well as from Synfuels China.

\section{References and recommended reading}

Papers of particular interest, published within the period of review, have been highlighted as:

- of special interest

-. of outstanding interest

1. Coppens MO: Multiscale nature inspired chemical engineering. In Multiscale Methods-Bridging the Q3Scales in Science and Engineering. Edited by Scales in Science and Engineering Oxford: Oxford University Press; 2009:536-560.

2. Mann S: Biomineralization: Principles and Concepts in

- Bioinorganic Materials and Chemistry. Oxford; 2001.

Excellent overview from a master in the field; see references below for more recent innovations and applications.

3. Ozin GA, Arsenault AC, Cademartiri L: Nanochemistry-A

- Chemical Approach to Nanomaterials. edn 2. Royal Society of Chemistry; 2009.

Beautiful overview of the magical world of nanomaterials, their properties and how to make them; includes plenty of historical background information, biomimetic approaches and biomineralization.

4. Tang Z, Wang Y, Podsiadlo P, Kotov NA: Biomedical applications of layer-by-layer assembly: from biomimetics to tissue engineering. Adv Mater 2006, 18:3203-3224.

5. Aizenberg J, Fratzl P: Biological and biomimetic materials. Adv Mater 2009, 21:387-388.

6. Kim Y-Y, Ganesan K, Yang P, Kulak AN, Borukhin S, Pechook S, Ribeiro L, Kröger R, Eichhorn SJ, Armes SP et al: : Artificial biominerals - incorporation of copolymer micelles in calcite single crystals. Nat Mater 2011, 10:890-896.

7. Que L, Tolman WB: Biologically inspired oxidation catalysis. Nature 2008, 455:333-340.

8. Marchetti L, Levine M: Biomimetic catalysis. ACS Catal 2011, 1:1090-1118.

9. Wulff G, Liu J: Design of biomimetic catalysts by molecular imprinting in synthetic polymers: the role of transition state stabilization. Acc Chem Res 2011 doi: 10.1021/ar200146m.

10. Ramaswamy S: One atom makes all the difference. Science 2011, 334:914-915.

11. Kalyanasundaram K, Graetzel M: Artificial photosynthesis:

- biomimetic approaches to solar energy conversion and storage. Curr Opin Biotechnol 2010, 21:298-310.

Excellent review on artificial photosynthesis by inventor of the dyesensitized solar cell, inspired by the mechanism of natural photosynthesis. 
12. Service RF: Turning over a new leaf. Science 2011, 334: - 925-927.

State-of-the-art on artificial photosynthesis.

13. Grätzel M: Recent advances in sensitized mesoscopic solar cells. Acc Chem Res 2009, 41:1788-1798.

14. Reece SY, Hamel JA, Sung K, Jarvi TD, Esswein AJ, Pijpers JJH, Nocera DG: Wireless solar water splitting using silicon-based semiconductors and earth-abundant catalysts. Science 2011, 334:645-648.

15. Kluwer AM, Kapre R, Hartl F, Lutz M, Spek AL, Brouwer AM, van Leeuwen PWNM, Reek JNH: Self-assembled biomimetic [2Fe2S]-hydrogenase-based photocatalyst for molecular hydrogen evolution. Proc Natl Acad Sci USA 2009, 106:1046010465.

16. Mandelbrot BB: The Fractal Geometry of Nature. revised and - updated edn. Freeman; 1983.

Q4 A masterpiece that opens our eyes to new symmetries in nature, capable of describing the rough, heterogeneous, and similar at multiple length scales; discover something new every time you open this book.

17. Weibel ER: Fractal geometry: a design principle for living organisms. Am J Physiol 1991, 261:L361-L369.

18. Vogel S: Glimpses of Creatures in Their Physical Worlds. Princeton - University Press; 2009.

Wonderful text on the mechanics and transport processes of living organisms, also published as a series of 12 articles in the $J$ Biosciences.

19. Coppens, M-O: Geometrical control of multiphase processes using a new fluid injection system. AlChE Symp Ser 1999, Paper 288c.

20. Kearney MM: Engineered fractals enhance process

- applications. Chem Eng Prog 2000, 96:61-68.

Introduces the concepts of area-filling and space-filling fractal distributors for mixing and separation processes, such as adsorption and chromatography.

21. Coppens M-O: Scaling up and down in a nature inspired way. - Ind Eng Chem Res 2005, 44:5011-5019.

Discusses mechanisms inspired by nature to increase the performance of multiphase reaction processes, with special focus on scalability.

22. Kjelstrup S, Coppens MO, Pharoah J, Pfeifer P: Nature-inspired energy and material efficient design of a polymer electrolyte membrane fuel cell. Energy Fuels 2010, 24:5097-5108.

23. Christensen DO, Nijenhuis J, van Ommen JR, Coppens M-O performance of a bubbling fluidized-bed reactor. Ind Eng Chem

Q5 Res 2008, 47:3601-3618. nature-inspired, fractal injector in fluidization (Figure 1, first column of images).

24. Van Ommen JR, Nijenhuis J, Coppens M-O: Reshaping the structure of fluidized beds. Chem Eng Prog 2009:49-57.

25. Murray CD: The physiological principle of minimum work. I. The vascular system and the cost of blood volume. Proc Natl Acad Sci USA 1926, 12:207-214.

26. Murray CD: The physiological principle of minimum work. II. Oxygen exchange in capillaries. Proc Natl Acad Sci USA 1926, 12:299-304.

27. West GB, Brown JH, Enquist BJ: A general model for the origin

- of allometric scaling laws in biology. Science 1997, 276:122126.

Compelling article on how the $3 / 4$ power law for metabolic rates could be explained by a space-filling fractal vascular network with minimal energy dissipation; note, however, that other interpretations have been provided.

28. West GB, Brown JH, Enquist BJ: The fourth dimension of life: fractal geometry and allometric scaling of organisms. Science 1999, 284:1677-1679.

29. West GB, Brown JH, Enquist BJ: A general model for the structure and allometry of plant vascular systems. Nature 1999, 400:664-667.

30. Bejan A: Shape and Structure, From Engineering to Nature.

-. Cambridge University Press; 2000.
- Influence of distributed secondary gas injection on the
Bejan provides a non-equilibrium thermodynamic basis for why trees are often optimal architectures in nature and engineering, and generalizes this in what he calls 'constructal theory'.

31. Bejan A, Lorente S: Design with Constructal Theory. Wiley; 2008.

32. Cho K-H, Lee J, Kim M-H, Bejan A: Vascular design of constructal structures with low flow resistance. Int $J$ Therm Sci 2010, 49:2309-2318.

33. Gheorghiu S, Kjelstrup S, Pfeifer P, Coppens M-O: Is the lung an optimal gas exchanger? In Fractals in Biology and Medicine, vol. IV. Edited by Nonnenmacher TF, Losa GA, Weibel ER. Birkhäuser: Springer Verlag; 2005:31-42.

34. Tondeur D, Luo L: Design and scaling laws of ramified fluid distributors by the constructal approach. Chem Eng Sci 2004 , 59:1799-1813.

35. Tondeur D, Fan Y, Luo L: Constructal optimization of arborescent structures with flow singularities. Chem Eng Sci 2009, 64:3968-3982.

36. Coppens M-O, Wang G: Optimal design of hierarchically structured porous catalysts. In Design of Heterogeneous Catalysts. Edited by Umit Ozkan. Wiley; 2009:25-58.

37. Sahimi M, Gavalas GR, Tsotsis TT: Statistical and continuum models of fluid solid reactions in porous media. Chem Eng Sci 1990, 45:1443-1502.

38. Salciccioli M, Stamatakis M, Caratzoulas S, Vlachos DG: A review of multiscale modeling of metal-catalyzed reactions: mechanism development for complexity and emergent behavior. Chem Eng Sci 2011, 66:4319-4355.

39. Keil FJ: Multiscale modeling in computational heterogeneous catalysis. Top Curr Chem 2012, 307:69-108.

40. Wang J, Groen JC, Yue W, Zhou W, Coppens M-O: Facile synthesis of ZSM-5 composites with hierarchical porosity. $J$ Mater Chem 2008, 18:468-474.

41. Pérez-Ramírez J, Abelló S, Bonilla A, Groen JC: Tailored mesoporosity development in zeolite crystals by partial detemplation and desilication. Adv Funct Mat 2009, 19:164-172.

42. Chal $R$, Gérardin $C$, Bulut $M$, van Donk S: Overview and industrial assessment of synthesis strategies towards zeolites with mesopores. ChemCatChem 2011, 3:67-81.

Recent, clear perspective on the synthesis of hierarchically structured zeolites, with unique discussion of industrial potential.

43. Johannessen E, Wang G, Coppens M-O: Optimal distributor networks in porous catalyst pellets. I. Molecular diffusion. Ind Eng Chem Res 2007, 46:4245-4256.

44. Wang G, Johannessen E, Kleijn CR, de Leeuw SW, Coppens M-O:

- Optimizing transport in nanostructured catalysts: a computational study. Chem Eng Sci 2007, 62:5110-5116.

Proof that introducing uniform macro/mesoporosity in nanoporous catalysts can drastically increase overall yield of diffusion limited reactions, but that a broad macro/mesopore size distribution is unnecessary.

45. Rao SM, Coppens M-O: Mitigating deactivation effects through rational design of hierarchically structured catalysts: application to hydrodemetalation. Ind Eng Chem Res 2010, 49:11087-11097.

46. Rao SM, Coppens M-O: Increasing robustness against - deactivation of nanoporous catalysts by introducing an optimized hierarchical pore network-application to hydrodemetalation. Chem Eng Sci 2012 http://dx.doi.org/ 10.1016/j.ces.2011.11.044.

An optimal uniform pore size and uniform distribution of porosity is very close to optimal in maintaining catalyst activity, even up to the reactor scale.

47. Wang G, Coppens MO: Rational design of hierarchically structured porous catalysts for autothermal reforming of methane. Chem Eng Sci 2010, 65:2344-2351.

48. Keil FJ, Rieckmann C: Optimization of three-dimensional catalyst pore structures. Chem Eng Sci 1994, 49:4811-4822.

49. Wei J: Catalyst designs to enhance diffusivity and performance-I: concepts and analysis. Chem Eng Sci 2011 , 66:4382-4388 
50. Van Gerven T, Stankiewicz A: Structure, energy, synergy, timethe fundamentals of process intensification. Ind Eng Chem Res 2009, 48:2465-2474.

51. Fratzl P, Weinkamer R: Nature's hierarchical materials. Prog Mater Sci 2007, 52:1263-1334.

52. Nam YS, Magyar AP, Lee D, Kim J-W, Yun DS, Park H, Pollom TS, Weitz DA, Belcher AM: Biologically templated photocatalytic nanostructures for sustained light-driven water oxidation. Nat Nanotechnol 2010, 5:340-344.

53. Mou J, Sheng S, Ho R, Shao Z: Chaperonins GroEL and GroES views from atomic force microscopy. Biophys $J$ 1996, 71:22132221

54. Tang Y-C, Chang H-C, Chakraborty K, Hartl FU, Hayer-Hartl M: Essential role of the chaperonin folding compartment in vivo. EMBO J 2008, 27:1458-1468.

55. Hartl FU, Hayer-Hartl M: Converging concepts of protein folding in vitro and in vivo. Nat Struct Mol Biol 2009, 16:575-581.

56. Hartl FU, Bracher A, Hayer-Hartl M: Molecular chaperones in - protein folding and proteostasis. Nature 2011, 475:324-332. Reviews the role of molecular chaperones, such as GroEL/GroES in $E$. coli, and how confinement affects protein folding in vivo.

57. Sang L-C, Coppens M-O: Effects of surface curvature and

- surface chemistry on the structure and function of proteins adsorbed in nanopores. Phys Chem Chem Phys 2011, 13:66896698

Demonstrates structural changes and tunability of catalytic activity of enzymes in mesopores, as a function of surface chemistry and pore diameter.

58. Tran DN, Balkus KJ: Perspective of recent progress in - immobilization of enzymes. ACS Catal 2011, 1:956-968

Excellent review of the state of the art on enzyme immobilization in mesoporous materials.

59. Cheung MS, Thirumalai D: Nanopore-protein interactions dramatically alter stability and yield of the native state in restricted spaces. J Mol Biol 2006, 357:632-643.

60. Rathore N, Knotts TA, de Pablo JJ: Confinement effects on the thermodynamics of protein folding: Monte Carlo simulations. Biophys J 2006, 90:1767-1773.

61. Mittal J, Best RB: Thermodynamics and kinetics of protein folding under confinement. Proc Natl Acad Sci USA 2008, 105:20233-20238

62. England J, Lucent D, Pande V: Rattling the cage: computational

- models of chaperonin-mediated protein folding. Curr Opin Struct Biol 2008, 18:163-169.

Reviews computational insights on the role of chaperonins.

63. Javidpour L, Tabar MRR, Sahimi M: Molecular simulation of protein dynamics in nanopores. I. Stability and folding. $J$ Chem Phys 2008, 128:115105.

64. Lucent D, Vishal, Pande VS: Protein folding under confinement: a role for solvent. Proc Natl Acad Sci USA 2007, 104:1043010434.

65. Tian JH, Garcia AE: Simulation studies of protein folding/ - unfolding equilibrium under polar and nonpolar confinement. $J$ Am Chem Soc 2011, 133:15157-15164.

Fully atomistic simulation opens the way to understanding steric, solvent, electrostatic and hydrophobic effects in biological and bioinspired channels, yet the systems are still small and coarse-grained simulations remain necessary for larger enzymes and wider pores.

66. Kauffman SA: The Origins of Order: Self-Organization and Selection in Evolution. Oxford University Press; 1993.

67. Goldenfeld N, Kadanoff LP: Simple lessons from complexity - Science 1999, 284:87-88.

Nice introduction to complexity and the physical challenges. Warns not to model bulldozers with quarks!

68. Ottino JM: Complex systems. AlChE J 2003, 49:292-

•• 299.

Perspective on the opportunities presented by the physics of complex systems for chemical engineering applications.
69. Grzybowski BA, Wilmer CE, Kim J, Browne KP, Bishop KJM: Self- assembly: from crystals to cells. Soft Matter 2009, 5:1110-1128. Clear, general tutorial on self-assembly.

70. Sheintuch M, Schmitz HA: Oscillations in catalytic reactions Cat Rev 1977, 15:107-172.

71. Ziff RM, Gulari R, Barshad Y: Kinetic phase transitions in an irreversible surface-reaction model. Phys Rev Lett 1986 , 56:2553-2556.

72. Imbihl R, Ertl G: Oscillatory kinetics in heterogeneous catalysis - Chem Rev 1995, 95:697-733.

Excellent overview of the topic.

73. Zhdanov VP: Monte Carlo simulations of oscillations, chaos and pattern formation in heterogeneous catalytic reactions. Surf Sci Rep 2002, 45:231-326.

74. Cross MC, Hohenberg PC: Pattern formation out of equilibrium

-. Rev Mod Phys 1993, 65:851-1112.

Exhaustive reference on pattern formation with numerous examples.

75. Melo F, Umbahowar P, Swinney HL: Transition to parametric wave patterns in a vertically oscillated granular layer. Phys Rev Lett 1994, 72:172-175.

76. Jaeger HM, Nagel SR, Behringer RP: Granular solids, liquids, and gases. Rev Mod Phys 1996, 68:1259-1273.

77. Aranson IS, Tsimring LS: Patterns and collective behavior in granular media: theoretical concepts. Rev Mod Phys 2006, 78:641-692.

78. Whitesides GM, Grzybowski B: Self-assembly at all scales. Science 2002, 295:2418-2421.

Classical paper on static and dynamic self-assembly.

79. Coppens MO, van Ommen JR: Structuring chaotic fluidized beds. Chem Eng J 2003, 96:117-124.

Discusses two novel ways to structure fluidized beds in a nature-inspired way: fractal injector and periodic pulsing.

80. van den Bleek CM, Coppens MO, Schouten JC: Application of chaos analysis to multiphase reactors. Chem Eng Sci 2002, 57:4763-4778.

81. Akhavan A, van Ommen JR, Nijenhuis J, Wang XS, Coppens M-O Rhodes M: Improved drying in a pulsation-assisted fluidized bed. Ind Eng Chem Res 2009, 48:302-309.

82. Hadi B, van Ommen JR, Coppens M-O: Enhanced particle mixing in pulsed fluidized beds, and the effect of internals. Ind Eng Chem Res 2011, in press, doi:10.1021/ie200933k [ASAP article].

83. Pence DV, Beasley DE: Chaos suppression in gas-solid fluidization. Chaos 1998, 8:514-519.

84. van Ommen JR, Nijenhuis J, van den Bleek CM, Coppens MO: Four ways to introduce structure in fluidized bed reactors. Ind Eng Chem Res 2007, 46:4236-4244.

85. Wang XS, Rhodes MJ: Pulsed fluidization - a DEM study of a fascinating phenomenon. Powder Technol 2005, 159:142-149.

86. Kiss IZ, Rusin CG, Kori H, Hudson JL: Engineering complex dynamical structures: sequential patterns and desynchronization. Science 2007, 316:1886-1889.

87. Shapiro JA: Bacteria are small but not stupid: cognition, natural - genetic engineering and socio-bacteriology. Stud Hist Phil Biol Biomed Sci 2007, 38:807-819.

Overview of bacterial communities as multi-cellular organisms by longtime expert in the field.

88. Matsuyama T, Harshey RM, Matsushita M: Self-similar colony morphogenesis by bacteria as the experimental model of fractal growth by a cell population. Fractals 1993, 1:302-311.

89. Ben-Jacob E: Social behavior of bacteria: from physics to complex organization. Eur Phys J B 2008, 65:315-322.

90. Vicsek T: Fractal Growth Phenomena. edn 2. World Scientific; 1992

91. Brenner K, You L, Arnold FH: Engineering microbial consortia: a new frontier in synthetic biology. Trends Biotechnol 2008 , 26:483-489. 
92. Huang Y, Krumanocker I, Coppens M-O: Fractal self-organization Q6 of bacteria-inspired agents. Fractals 2012, submitted for publication [Special Issue in memory of Benoit Mandelbrot]

93. Schweitzer F: Brownian Agents and Active Particles: Collective - Dynamics in the Natural and Social Sciences. Springer; 2003. Excellent introduction of agent-based systems, with plenty of references and examples.

94. Luisi PL: The Emergence of Life: From Chemical Origins to Synthetic Biology. Cambridge University Press; 2006.

95. Stano P, Luisi PL: Achievements and open questions in the self- reproduction of vesicles and synthetic minimal cells. Chem Commun 2010, 46:3639-3653.

Status on the synthesis of dividing vesicles that could be filled with a biomolecular cargo to produce artificial cells.

96. Watts DJ, Strogatz SH: Collective dynamics of "small world" networks. Nature 1998, 393:440-442.

97. Albert R, Barabási AL: Statistical mechanics of complex networks. Rev Mod Phys 2002, 74:47-97.

98. Amaral LAN, Ottino JM: Complex systems and networkschallenges and opportunities for chemical and biological systems. Chem Eng Sci 2004, 59:1653-1666.
99. Venkatasubramanian V, Katare S, Patkar P, Mu F-P: Spontaneous emergence of complex optimal networks through evolutionary adaptation. Comput Chem Eng 2004, 28:1789-1798.

100. Katare S, West DH: Optimal complex networks spontaneously emerge when information transfer is maximized at least expense: a design perspective. Complexity 2006, 11:26-35.

101. He J, Sun J, Deem MW: Spontaneous emergence of modularity - in a model of evolving individuals and in real networks. Phys Rev E 2009, 79:031907.

Shows how modularity (relatively independent, clustered elements of hierarchy) commonly arises in biology, in response to a changing envir onment.

102. Sang LC: PhD Thesis, Rensselaer Polytechnic Institute.

103. In: http://classes.yale.edu/fractals/IMA//FB/NatFrac/Lung1.gif.

104. B Cioc: Photograph of leaf provided by S Gheorghiu.

105. Ben-Jacob E, Shochet O, Tenenbaum A, Cohen I, Czirók A Vicsek T: Communication, regulation and control during complex patterning of bacterial colonies. Fractals 1994, 2:15-44. 